\title{
Correlation between BASDAI and ASDAS to evaluate the activity of patients with spondyloarthritis
}

\author{
H. SAHRAOUI, S. AIT MALEK, A. MOUGUI, S. EL RHARRAS, I. EL BOUCHTI \\ Department of rheumatology. Mohamed VI university hospital; Marrakech; Morocco
}

\section{INTRODUCTION}

Ankylosing Spondylitis Disease Activity Score (ASDAS) is a new measurement tool to assess disease activity in patients with spondyloarthritis (SPA). It was developed because of BASDAI's limitation for being totally patient-derived with limited validity. The aim of the study was to compare ASDAS with BASDAI for evaluation of spondyloarthritis activity in our department.

\section{MATERIELS AND METHODES}

- We conducted a descriptive and comparatif study in the university center of Marrakech over 10 years from January 2007 to Novembre 2017.

- We included cases of spondyloarthritis fulfilling ASDAS or AMOR classification criteria. The disease activity assessed by BASDAI and ASDAS-CRP should be evaluate at the same visit.

- The demographic characteristics were collected as well as clinical and biological characteristics.

- The IBM SPSS statistics was used for statistical documentation.

- The correlation between ASDAS and BASDAI was evaluated using Pearson's correlation coefficients (r, $p$ values). Statically significant covariate was considered if $\mathrm{p}<0.05$.

\section{RESULTATS}

-Among 84 cases, the mean age was 40.8+/-14.2 years, with a male predominance in $60.7 \%$ of the cases.

-The SPA was a pure axial form in 6 patients (7.14\%), association of axial, peripheral and enthesic form was in 35 cases $(41.6 \%)$.

- The mean BASDAI was $4.51+/-1,8$ and the mean ASDAS was $2.77+/-$ 1.1 .

-A very strong positive correlation was found between BASDAI and $\operatorname{ASDAS}(\mathrm{r}=0.33 \mathrm{P}<0.005)$.

- Thirty eight percent of patients had an inactive disease according to BASDAI whereas $14.3 \%$ had an inactive disease according to ASDAS.

- Among patients who had a high disease activity according to BASDAI, 2 patients were inactive according to ASDAS score $(3.8 \%), 12$ patients had a moderate activity $(23.1 \%), 19$ patients had a high activity (36.5\%) and 19 others had a very high activity (36.5\%). - In general, BASDAI values are directly related to ASDAS values (p: 0.004). Patients with inactive disease according to BASDAI had a higher mean ASDAS than patients with inactive disease according to BASDAI (3.07 \pm 1.08 and $2.28 \pm 1.09$, respectively, p: 0.002). )

Références:

1. Lihi Eder. et al. Is ASDAS better than BASDAI as a measure of disease activity in axia psoriatic arthritis? Ann Rheum Dis 2010;69:2160-2164.

G. Kilic et al. Is there any gender-specific difference in the cut-off values of ankylosing spondylitis disease activity score in patients with axial spondyloarthritis? International Journal of Rheumatic Diseases 2016

3. G. K1lıç. et al. Comparison of ASDAS and BASDAI as a measure of disease activity in axial psoriatic arthritis. Clin Rheumatol; 2014.
- The correlation of disease activity categories expressed in BASDAI with the ASDAS values showed that this correlation is more significant in the active SPA group (BASDAI), there were only two patients who had active disease according to BASDAI and not according to ASDAS. -In the inactive SPA group (BASDAI), however, $70 \%$ of the patients had active disease according to the ASDAS (see table below)

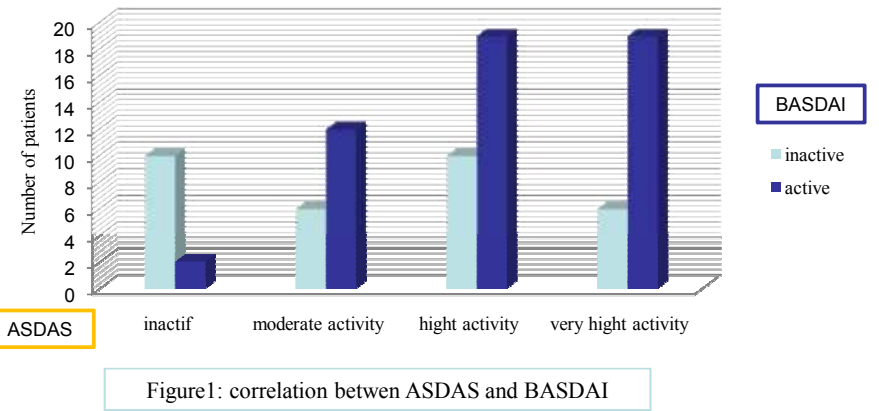

\section{DISCUSSION}

In this study we compared two scores for assessment of disease activity in patients with spondyloarthritis : the BASDAI and the newly developed ASDAS.

Both scores showed good correlation with the construct of disease activity when based on patient reported scores.

The BASDAI is still a widely used disease activity tool which relies only on patient-reported data; therefore, the need for a more objective disease activity measure for the use in spondyloarthritis is evident. ASDAS is a new index which combines the objective (inflammatory markers including ESR and/or CRP) and subjective disease activity measures (patient's self-assessment). ASDAS has been also proved to be a valuable tool for monitoring the treatment. It was recently shown that ASDAS performs better than BASDAI in evaluating disease activity in patients with spondyloarthritis

Patients with axial spondyloarthritis may have coexisting conditions like fibromyalgia (FM) which makes patient assessment for disease activity and functional impairment much more complicated. Salaffi et al. Showed that fibromyalgia is common in axial spondyloarthritis and ASDAS is better than BASDAI to distinguish patients with disease activity from those having functional impairment.

\section{CONCLUSION}

Our study suggests that ASDAS and BASDAI scores have a good discriminative ability and correlation. However, multiple studies showed that the use of the ASDAS-CRP index is more appropriate for a more pertinent evaluation of disease activity. 